\title{
The potential utility of real-time PCR of the 16S-rRNA gene in the diagnosis of neonatal sepsis
}

\author{
Kenan İstanbullu ${ }^{1}$, Nilgün Köksal², Merih Çetinkaya ${ }^{3}$, Hilal Özkan², Tahsin Yakut ${ }^{3}$, \\ Mutlu Karkucak ${ }^{3}$, Haldun Doğan ${ }^{4}$ \\ Departments of ${ }^{1}$ Pediatrics, ${ }^{2}$ Neonatology, and ${ }^{4}$ Medical Genetics, Uludağ University Faculty of Medicine, Bursa; \\ ${ }^{3}$ Department of Neonatology, Kanuni Sultan Suleyman Training and Research Hospital, İstanbul; ${ }^{4}$ Department of Intergen \\ Genetics Center, Ankara, Turkey, E-mail: hiozkan@hotmail.com \\ Received: 31st July 2018, Revised: 26th October 2018, 3rd January 2019, Accepted: 29th January 2019
}

SUMMARY: İstanbullu K, Köksal N, Çetinkaya M, Özkan H, Yakut T, Karkucak M, Doğan H. The potential utility of real-time PCR of the 16S-rRNA gene in the diagnosis of neonatal sepsis. Turk J Pediatr 2019; 61: 493-499.

The purpose of this study was to evaluate the efficacy of real-time polymerase chain reaction (PCR) of the 16S rRNA gene in diagnosis of neonatal sepsis and compare it with conventional blood culture.

A total of 150 infants were enrolled in this prospective study. The infants were classified into two groups: sepsis group $(n=100)$ and control group $(n=50)$. Blood samples for complete blood count, C-reactive protein, procalcitonin, serum-amyloid A, blood culture and PCR were obtained before initiating antibiotic treatment. Eight specific probes were used to perform PCR analysis for detection of 8 different microorganisms.

The positivity rates of blood culture and PCR were found as $11 \%$ and $3 \%$, respectively. The diagnosis of neonatal sepsis by PCR revealed a $16.6 \%$ sensitivity, $97.8 \%$ specificity, $33.3 \%$ positive predictive value and $94.8 \%$ negative predictive value compared with the blood culture.

This study showed a low sensitivity of PCR of the 16S rRNA gene in the diagnosis of neonatal sepsis. This may be associated with the identification of rare microorganisms in the blood culture that were not included to PCR analysis. Implementation of all suspectible microorganisms into PCR assay may increase the sensitivity of $16 \mathrm{~S}$ rRNA gene PCR in diagnosis of neonatal sepsis.

Key words: $16 S$ rRNA, blood culture, PCR, neonatal sepsis, newborn.

Neonatal sepsis still represents an important cause of mortality and morbidity among infants, as almost one million newborns die from infections every year mostly in low-income countries. ${ }^{1}$ The incidence of neonatal sepsis varies between 1-5/1000 to 49-170/1000 live births, and is especially more frequent in very low birth weight (VLBW) infants., ${ }^{2,3}$ Despite important advances in perinatal and neonatal care, neonatal sepsis remains a major problem throughout the world. The high incidence and severe outcome of neonatal sepsis are mainly related to the combination of the reduced immune defence and the complex interactions between the microorganism and the host response. ${ }^{4}$

Although bacteria and viruses are the most frequent causative agents, fungi and parasites also play an important role in neonatal sepsis etiology. ${ }^{5}$ However, the diagnosis is difficult as infants have generally subtle signs and symptoms and several laboratory methods lack in both sensitivity and specificity. ${ }^{6}$ Early signs and symptoms of neonatal sepsis may easily be confused with non-infectious conditions which results with unnecessarily hospitalization and administration of antibiotics in these infants. 
To date, blood culture is still considered as the gold standard method for diagnosis of neonatal sepsis. However, it has some limitations such as low sensitivity and the long time it takes for the results which may delay antibiotic therapy and compromise the life of newborns. ${ }^{7}$

In recent years, molecular techniques including polymerase chain reaction (PCR) was reported to be used successfully in the diagnosis of neonatal sepsis by identification of specific bacterial, fungal and viral genes through amplification of target DNA/RNA fragments. ${ }^{8}$ The amplification of 16S rRNA gene with broad range PCR analysis was found to have $100 \%$ sensitivity, $95 \%$ specificity, $77 \%$ positive predictive value (PPV), and $100 \%$ negative predictive value (NPV) as compared to blood culture. ${ }^{9}$

The aim of this study was to determine the utility of real time PCR in the diagnosis of neonatal sepsis and also to compare it with conventional blood culture.

\section{Material and Methods}

This prospective study was performed on infants with clinical and laboratory findings of neonatal sepsis who were admitted to Neonatal Intensive Care Unit (NICU). During the study period, a total of 362 infants were hospitalized and assessed for the eligibility for this study. From these, a total of 100 infants were diagnosed with high probable neonatal sepsis according the criteria defined by Gitto et al. ${ }^{10}$ The study group consisted of these neonates who were clinically suspected to have either early or late onset sepsis. A total of 50 neonates with no signs of clinical and laboratory infection constituted the control group. As the primary objective of this study was to evaluate the utility of 16S RNA PCR analysis in the diagnosis of neonatal sepsis, both infants with and without clinical/ laboratory findings were included. The secondary objective was to compare the effectiveness of PCR with conventional blood culture. However, as the number of infants admitted to our NICU without clinical/ laboratory findings were limited, the study groups consisted of 100 vs 50 infants in the control group. The study protocol was approved by the Uludag University Faculty of Medicine Ethics Committee at 23 June 2009 date and 2009-12/36 number. The financial source of the study was Uludag University Scientific Research Project. The study was performed between December 2010 and July 2012.

Informed parental consent was obtained for all infants. All inborn and outborn, preterm and term hospitalized infants during the first 28 days of life, either with early/late sepsis or other conditions such as hyperbilirubinemia and hypoglycemia were included. Exclusion criteria included administration of antibiotic therapy at admission, lack of laboratory data (infants whose blood culture or PCR analysis resulted as contamination) and refusal of parental consent. Although 362 neonates were assesed for the eligibility, 84 infants had been on antibiotic therapy at admission, 11 infants had lack of laboratory data and 117 parents did not give consent so the final group consisted of 150 infants. A total of 18 infants died during the study period.

Both maternal and demographical data including maternal infection, presence of preterm premature rupture of membranes (PPROM) and/or chorioamnionitis, preeclampsia, birth weight, gestational age, gender, prematurity, and mode of delivery were all recorded. Early sepsis was defined as sepsis with onset $\leq 3$ days of age and late sepsis was defined as occuring greater than 3 days after delivery. Temperature instability, apnea, need for supplemented oxygen, need for ventilation, tachycardia/ bradycardia, hypotension, jaundice (increased serum total bilirubin levels according to postnatal age), feeding intolerance (either gastric residuals and/or emesis $>50 \%$ of previous volume), abdominal distension, necrotizing enterocolitis were considered as the clinical signs of sepsis. Changes in hematologic parameters were processed according to the Manroe and Rodwell scoring system. ${ }^{11,12}$ Leukopenia was defined as leukocyte counts $\leq 5,000 / \mathrm{mm}^{3}$; leukocytosis was defined as leukocyte counts $\geq 25,000 / \mathrm{mm}^{3}$ at birth, $\geq 30,000 / \mathrm{mm}^{3}$ at 12 24 hours and $\geq 21,000 / \mathrm{mm}^{3}$ after the second day. Thrombocytopenia was defined as platelet counts $\leq 150,000 / \mathrm{mm}^{3}$. 
A prospective sepsis screen including total leukocyte count, absolute neutrophil count, immature-to-total neutrophil ratio, blood smear evaluation, C-reactive protein (CRP), procalcitonin (PCT) and serum amyloid A (SAA) levels was performed in all infants with probable sepsis. Once the infant was diagnosed with neonatal sepsis according to the sepsis screening, two seperate sets of blood cultures from two different sites were obtained before initiating the antibiotic therapy. An additional 0.5-1 mL EDTA-blood sample was also collected for 16S rRNA gene PCR amplification.

For blood culture processing, an automated continous-monitoring blood culture system, BACTEC 9240 (Becton Dickinson, Sparks, MD, USA) was used. A minimum of $1 \mathrm{~mL}$ blood was added into the blood culture bottle. The bottles were sent to the microbiology laboratory and were incubated immediately in the laboratory according to the manufacturer's instructions. The EDTA-blood samples for PCR were stored at $+2^{\circ} /+8^{\circ} \mathrm{C}$ before analysis and all samples collected for PCR were studied at once after completion of study. DNA was prepared from all samples by using Realpure Spin Kit (Durviz SLU, Spain). The protocol for bacterial DNA isolation from biological fluids was used. ${ }^{13}$ In this study, the DNA of selected bacteria was detected by using Real-Time PCR method and hydrolysis probes. Primers designed to amplify two regions in $16 \mathrm{~S}$ rRNA genes of the selected bacteria (Enterococcus spp., Escherichia coli, Klebsiella pneumoniae, Streptococcus agalactiae, Haemophilus influenzae, Listeria monocytogenes, Staphylococcus epidermidis, and Staphylococcus aureus) are used together with species specific probes. The reaction condition included an activation step at $95^{\circ} \mathrm{C}$ for $10 \mathrm{~min}$ followed by 50 cycles of $95^{\circ} \mathrm{C}$ for 15 $\mathrm{sec}, 58^{\circ} \mathrm{C}$ for $45 \mathrm{sec}$ and $72^{\circ} \mathrm{C}$ for $20 \mathrm{sec}$. During PCR, Taq polymerase hydrolysis the probes, releasing the fluorescent dye attached to the probes to result an increase in the amount of florescence. The detection of the species is achieved by the analysis of the signals acquired during Rotor-Gene ${ }^{\mathrm{TM}} 6000$ Real-Time PCR (Qiagen N.V.). The PCR technique is a rapid technique that results within 3 hours to 16 hours including sampling, extraction and assay time. ${ }^{14,15}$
SPSS software (SPSS, version 20.0, Chicago, IL, USA) was used for statistical analyses. Descriptive statistics were given as mean, median, minimum, maximum and percentage. Mann-Whitney test, Pearson's chi-square test, Fisher's test and McNemar's test were used for comparisons between groups as appropriate. Values of $\mathrm{p}<0.05$ were considered to be significant.

\section{Results}

A total of 150 infants were enrolled in this study. There were 100 infants in the sepsis group and 50 infants in the control group. The mean birth weight of the infants in

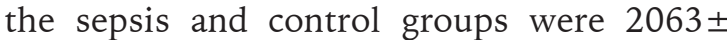
942 grams (min-max, 640 and $4090 \mathrm{~g}$ ) and $2776 \pm 668$ grams (min-max, 1780 and 4000 $\mathrm{g})$, respectively. The mean gestational age of the infants in sepsis and control groups were $34 \pm 4.5$ weeks (min-max, 24 to 41 weeks) and $37 \pm 1.8$ weeks (min-max, 35 to 41 weeks), respectively. The demographic and clinical characteristics of both sepsis and control groups are shown in Table I.

The most common findings of the infants in the sepsis group were tachypnea (66\%), respiratory distress $(50 \%)$, feeding intolerance $(50 \%)$, low activity (19\%), and jaundice (15\%). The levels of CRP, PCT and SAA at the time of diagnosis and 48 hours were significantly higher in the sepsis group compared with the control group. The positivity rates of blood culture and PCR were $11 \%$ and $3 \%$, respectively. Only 1 patient had a positive result for both blood culture and PCR. Of the cases with a positive blood culture six had early onset and 5 had late onset sepsis. There were two cases with late onset sepsis and 1 case with early onset sepsis in PCR positive group. The isolated pathogens from the blood culture were Staphylococcus capitis (2 infants), Staphylococcus epidermidis (4 infants), Staphylococcus haemolyticus, Streptococcus uberis, Escherichia coli (2 infants) and Candida parapsilosis. PCR analysis was positive for Escherichia coli and Enterococcus spp. In the third positive case, PCR could not make a differentiation between three signals including Klebsiella pneumonia, Enterococcus spp. and Streptococcus agalactiae. Therefore, Escherichia coli was the only bacteria that was 
Table I. Comparison of Maternal and Neonatal Demographic Data Between Sepsis and Control Groups.

\begin{tabular}{lccc}
\hline Study Population & $\begin{array}{c}\text { Sepsis group } \\
\mathrm{n}=100\end{array}$ & $\begin{array}{c}\text { Control group } \\
\mathrm{n}=50\end{array}$ & p value \\
\hline Maternal demographics & & & - \\
*Maternal infection, n (\%) & $7(4.6)$ & - & - \\
Premature rupture membranes, (\%) & $21(21)$ & - & - \\
Chorioamnionitis & $4(4)$ & - & 0.78 \\
Preeclampsia, n (\%) & $10(10)$ & $6(12)$ & \\
Neonatal demographics & & & 0.0001 \\
Birth weight (g), mean \pm std & $2063 \pm 942$ & $2776 \pm 668$ & 0.0001 \\
Gestational age (week), mean \pm std & $34 \pm 4.5$ & $37 \pm 1.6$ & 0.30 \\
Male gender, $\mathrm{n}(\%)$ & $59(59)$ & $25(50)$ & 0.009 \\
Prematurity (<37 weeks of gestation), $\mathrm{n}(\%)$ & $61(61)$ & $19(38)$ & 0.5 \\
Prematurity (<32 weeks of gestation) & $12(12)$ & $4(8)$ & 0.07 \\
Cesarian section, $\mathrm{n}(\%)$ & $64(64)$ & $24(48)$ & \\
\hline
\end{tabular}

*Maternal infection; the vaginal swab culture identified E. Coli in 3 mothers, K. Pneumonia in 2 mothers, E. Cloacae in 1 mother and $S$. aureus in 1 mother.

Table II. The Microorganisms Isolated from Blood Culture and PCR.

\begin{tabular}{lccc}
\hline Blood culture positive & $\mathrm{n}$ & PCR positive & $\mathrm{n}$ \\
\hline Staphylococcus epidermidis & 4 & Enterococcus faecalis & 2 \\
Staphylococcus capitis & 2 & Escherichia coli & 1 \\
Escherichia coli & 2 & & \\
Staphylococcus haemolyticus & 1 & & \\
Streptococcus uberis & 1 & & 3 \\
Candida parapsilosis & 1 & & \\
\hline Total & 11 & & \\
\hline
\end{tabular}

Table III. Comparison of Blood Culture and 16S rRNA PCR Results.

\begin{tabular}{lccc}
\hline & & Blood Culture & \\
\cline { 2 - 4 } & Positive & Negative & Total \\
\hline PCR & 1 & & \\
Positive & 5 & 2 & 3 \\
Negative & 6 & 92 & 97 \\
Total & 94 & 100 \\
\hline
\end{tabular}

PCR sensitivity $16.6 \%$, specificity $97.8 \%$, positive predictive value $33.3 \%$, negative predictive value $94.8 \%$.

detected in both blood culture and PCR analysis (Table II). During data collection, 11 infants (8 blood culture, 3 PCR) had results that were thought to be contamination, so these cases were excluded from the final analysis.
As PCR detected only 1 organism out of 6 potentially detectable bacteria, the diagnosis of neonatal sepsis by PCR revealed a $16.6 \%$ sensitivity, $97.8 \%$ specificity, $33.3 \%$ PPV and $94.8 \%$ NPV compared with blood culture in this selected PCR analysis, (Table III). 


\section{Discussion}

As neonatal sepsis may show rapid progression to multiple organ dysfunction syndrome and death, early diagnosis is very important. However, it is also difficult to diagnose neonatal sepsis as the clinical signs of sepsis may be similar to other life-threatining diseases such as necrotizing enterocolitis, respiratory distress syndrome and perinatal asphyxi. ${ }^{16}$ Although several biomarkers have been suggested to be used for the diagnosis of neonatal sepsis, automated blood culture systems have long been considered as the gold standard method for diagnosis. Despite improvements in instrumentation and growth media, results of blood culture may be delayed up to 48 hours. ${ }^{17}$ Bacterial load may also be low due to maternal antibiotic administration. Contamination rate in blood culture in neonates are also very high due to techical difficulties during the sterile venipuncture process. It is also difficult to interpret the identification of Coagulase-negative staphylococci in blood culture as these organisms can be both part of the normal skin flora or they may be pathogenic in these high risk infants. Blood culture has also been reported to be positive in only $5-10 \%$ of suspected neonatal sepsis cases. ${ }^{16,18}$ All these diagnostic difficulties lead to a greater use of broad spectrum antibiotics that in turn may cause prolonged and unnecessary hospitalization. These also promote antibiotic drug resistance in these infants. ${ }^{14}$ Therefore, newer rapid and accurate diagnostic tests are required to provide positive results in a timely manner in neonatal sepsis.

In recent years, real time PCR has been reported to be used succesfully by detecting a variety of microorganisms from sterile body sites in neonatal sepsis..$^{9,14,19-22}$ The advantages of molecular diagnostic tests such as PCR in neonatal sepsis include the ability to detect and amplify small amounts of bacterial DNA and also the rapid turnover time. A broad-range bacterial PCR test may also have an ability to diagnose neonatal sepsis in culture negative infants with low density bacteremia as the high sensivity of PCR allows bacterial DNA detection even low concentrations. ${ }^{16,23}$ The use of probe-based specific PCR was reported to reduce diagnostic time, help in identifying bacterial species and improve specificity and positive predictive value in neonatal sepsis. ${ }^{24}$ Real time PCR system is faster and associated with lower contamination rates as amplification and detection both occur simultaneously in a closed system. ${ }^{25}$ Therefore, we performed this prospective study to compare the utility of real-time PCR of the 16S rRNA gene with conventional blood culture in the diagnosis of neonatal sepsis. Although increasing number of studies have been published that evaluates the role of PCR techniques in the diagnosis of neonatal sepsis, varying success including high specificity and low sensivity (40\%) was reported. ${ }^{14,26}$ In a meta-analysis that evaluated the utility of molecular assays for the diagnosis of neonatal sepsis, the mean sensitivity and specificity were determined as 0.90 (95\% CI: 0.78-0.95) and 0.96 (95\% CI: 0.94-0.97), respectively. In this meta-analysis, PCR and broad-range conventional PCR were found to have higher sensitivity and specificity compared with other assays. ${ }^{27}$ Although some studies reported higher sensitivity, specificity and PPV values ${ }^{9,25}$, we found a lower sensitivity and PPV for PCR. However, our results were in accordance with some of the studies that yielded similar results. ${ }^{21,22}$ One of the main disadvantages of PCR analysis is the cost for implementing these technologies, including expenses for equipment, reagents, and personnel. As we had limited funding for the study, we used probes for the most common organisms responsible from neonatal sepsis. Unfortunately, blood culture was positive for different and rare organisms, the lower rates of PCR in our study may be associated with this issue. This point may be considered as the main limitation of our study. However, it may also be important for future similar studies to include more number and types of organisms to yield better results with PCR.

The main problem with real-time PCR testing is that the blood sample must be collected with a sterile venipuncture, which is generally difficult in neonates. There is also a high risk for contamination of enrichment media, reagents, or sample collection and processing. Some amount of contamination either from skin or laboratory environment were reported as an important limitation issue of PCR analysis in infants and in accordance, we had to exclude 3 infants due to contaminated PCR 
results. ${ }^{17,19}$ If the white cell count is high, low sensitivity may occur due to human DNA competition from human DNA in whole blood. For DNA analysis, bacterial organism requires lysis, however, it is difficult for Gram positive bacteria to lyse as they have resilient cell walls. ${ }^{16,26}$ Another limitation of the PCR technique is the lack of the availability of all existing genetic sequences for microbiota. In this study, although we studied the most common bacteria, rare pathogens such as Staphylococcus capitis and Streptococcus uberis were identified in the blood culture. In our PCR analysis, we included Staphylococcus epidermidis and Staphylococcus aureus as the most common gram positive microorganisms. Candida albicans growth occurred in one of the blood cultures, however we did not include any fungus to PCR analysis. Therefore, we suggest that a broad range of different bacteria must be used for PCR analysis. However, this will also lead to increased cost and will make PCR analysis more expensive.

Although some studies suggested the use of PCR method for quickly detecting microorganisms in patients with highly suspected sepsis and negative blood culture, this technology needs to be improved for better accuracy. According to the results from the meta-analysis by Pammi et $\mathrm{al}^{27}$ the authors reported that molecular techniques including 16S rRNA PCR analysis did not have sufficient sensitivity to replace blood culture in the diagnosis of neonatal sepsis. However, the authors recommended to perform these assays as "add-on" tests. ${ }^{27}$ In another review, molecular techniques were suggested to improve the diagnosis of neonatal sepsis, however, the authors established that the technology did not reach to routine clinical use of these assays. ${ }^{28}$ Our results are in accordance with these comments. We suggest performing PCR analysis that includes all the possible microorganisms that are responsible from neonatal sepsis.

Molecular techniques still have some limitations and disadvantages. The costs, availability of equipment and technical skills in the microbiological laboratory should be considered before routine clinical usage. The positive results of these molecular methods in the face of a negative blood culture should be interpreted very carefully in a clinical setting and sample contamination may have to be excluded as mentioned above. It must be kept in mind that false-negative results of molecular assays may be associated with inefficient DNA extraction, presence of low levels of pathogen DNA or the presence of inhibitors. Additionally, PCR analyses do not provide antibiotic sensitivity results that is very important for the antibiotic treatment duration in infants with neonatal sepsis. ${ }^{28}$

In conclusion, this study showed a low sensitivity and PPV of PCR of the 16S rRNA gene in the diagnosis of neonatal sepsis compared with conventional blood culture. This low sensitivity may be associated with the identification of rare microorganisms in the blood culture that were not included in the PCR analysis. Therefore, we suggest selecting a broad spectrum of microorganisms for PCR assay to increase the sensitivity of $16 \mathrm{~S}$ rRNA gene PCR in the diagnosis of neonatal sepsis. Until the use of PCR has been increased in neonates, it must be used in addition to conventional blood culture.

\section{Acknowleadgment}

The study was funded by Uludag University Scientific Research Project Council (ID: $\operatorname{UAP}(T)-2010 / 3$ )

\section{REFERENCES}

1. Zaidi AK, Ganatra HA, Syed S, et al. Effect of case management on neonatal mortality due to sepsis and pneumonia. BMC Public Health 2011; 11(Suppl 3): S13.

2. Shah BA, Padbury JF. Neonatal sepsis: An old problem with new insights. Virulence 2014; 5: 170-178.

3. Lawn JE, Cousens S, Zupan J; Lancet Neonatal Survival Steering Team. 4 million neonatal deaths: When? Where? Why? Lancet 2005; 365: 891-900.

4. Mussap M. Laboratory medicine in neonatal sepsis and inflammation. J Matern Fetal Neonatal Med 2012; 25(Suppl 4): 32-34.

5. Satar M, Ozlu F. Neonatal sepsis: a continuing disease burden. Turk J Pediatr 2012; 54: 449-457.

6. Malik A, Hui CP, Pennie RA, Kirpalani H. Beyond the complete blood cell count and C-reactive protein: a systematic review of modern diagnostic tests for neonatal sepsis. Arch Pediatr Adolesc Med 2003; 157: 511-516. 
7. Guerti K, Devos H, Ieven MM, Mahieu LM. Time to positivity of neonatal blood cultures: fast and furious? J Med Microbiol 2011; 60(Pt 4): 446-453.

8. McCabe KM, Khan G, Zhang YH, Mason EO, McCabe ER. Amplification of bacterial DNA using highly conserved sequences: automated analysis and potential for molecular triage of sepsis. Pediatrics 1995; 95: 165-169.

9. Liu C, Ai HW, Wang WP, et al. Comparison of $16 \mathrm{~S}$ rRNA gene PCR and blood culture for diagnosis of neonatal sepsis. Arch Pediatr 2014; 21: 162-169.

10. Gitto E, Karbownik M, Reiter RJ, et al. Effects of melatonin treatment in septic newborns. Pediatr Res 2001; 50: 756-760.

11. Manroe BL, Weinberg AG, Rosenfeld CR, Browne $\mathrm{R}$. The neonatal blood count in health and disease. I. Reference values for neutrophilic cells. J Pediatr 1979; 95: 89-98.

12. Rodwell RL, Leslie AL, Tudehope DI. Early diagnosis of neonatal sepsis using a hematological scoring system. J Pediatr 1988; 112: 761-767.

13. Jordan JA, Durso MB. Real-time polymerase chain reaction for detecting bacterial DNA directly from blood of neonates being evaluated for sepsis. J Mol Diagn 2005; 7: 575-581.

14. Jordan JA. Molecular diagnosis of neonatal sepsis. Clin Perinatol 2010; 37: 411-419.

15. Midan DA, Abo El Fotoh WMM, El Shalakany AH. The potential role of incorporating real-time PCR and DNA sequencing for amplification and detection of 16S rRNA gene signatures in neonatal sepsis. J Matern Fetal Neonatal Med 2017; 30: 1476-1483.

16. Edmond K, Zaidi A. New approaches to preventing, diagnosing and treating neonatal sepsis. PLoS Med 2010; 7: e1000213.

17. Kurlat I, Stoll BJ, McGowan JE Jr. Time to positivity for detection of bacteremia in neonates. J Clin Microbiol 1989; 27: 1068-1071.

18. Darmstadt GL, Batra M, Zaidi AK. Parenteral antibiotics for the treatment of serious neonatal bacterial infections in developing country settings. Pediatr Infect Dis J 2009; 28 (Suppl 1): 37-42.
19. Golden SM, Stamilio DM, Faux BM, et al. Evaluation of a real-time fluorescent PCR assay for rapid detection of group B Streptococci in neonatal blood. Diag Microbiol Infect Dis 2004; 50: 7-13.

20. Shang $S$, Chen Z, Yu X. Detection of bacterial DNA by PCR and reverse hybridization in the 16S rRNA gene with particular reference to neonatal septicemia. Acta Paediatr 2001; 90: 179-183.

21. Ohlin A, Bäckman A, Ewald U, Schollin J, Björkqvist M. Diagnosis of neonaal sepsis by broad range $16 \mathrm{~S}$ real-time polymerase chain reaction. Neonatology 2012; 101: 241-246.

22. Reier-Nilsen T, Farstad T, Nakstad B, Lauvrak V, Steinbakk M. Comparison of broad range 16S rRNA $\mathrm{CR}$ and conventional blood culture for diagnosis of sepsis in the newborn: a case control study. BMC Pediatr 2009; 9: 5.

23. Srinivasan L, Harris MC. New technologies for the rapid diagnosis of neonatal sepsis. Curr Opin Pediatr 2012; 24: 165-171.

24. Wu YD, Chen LH, Wu XJ, et al. Gram stain-specific probe-based real-time PCR for diagnosis and discrimination of bacterial neonatal sepsis. J Clin Microbiol 2008; 46: 2613-2619.

25. Jordan JA, Durso MB. Comparison of $16 \mathrm{~S}$ rRNA gene PCR and BACTEC 9240 for detection of neonatal bacteremia. J Clin Microbiol 2000; 38: 2574-2578.

26. Jordan JA, Durso MB, Butchko AR, Jones JG, Brozanski BS. Evaluating the near term infant for early onset sepsis: progress and challanges to consider with $16 \mathrm{~S}$ rDNA polymerase chain reaction testing. J Mol Diagn 2006; 8: 357-363.

27. Pammi M, Flores A, Leeflang $M$, Versalovic J. Molecular assays in the diagnosis of neonatal sepsis: a systematic review and meta-analysis. Pediatrics 2011; 128: e973-e985.

28. Venkatesh M, Flores A, Luna RA, Versalovic J. Molecular microbiological methods in the diagnosis of neonatal sepsis. Expert Rev Anti Infect Ther 2010; 8: 1037-1048. 